\title{
Investigator Name
}

National Cancer Institute

\section{Source}

National Cancer Institute. Investigator Name. NCI Thesaurus. Code C83302.

The literal identifier of an investigator. 